\title{
Unveiling the origin of oxygen atomic impurities in Au nanowires
}

\author{
A. P. F. Nascimento, ${ }^{1}$ Miguel A. San-Miguel, ${ }^{2}$ and E. Z. da Silva ${ }^{1}$ \\ ${ }^{1}$ Institute of Physics “Gleb Wataghin," University of Campinas-Unicamp, 13083-970 Campinas, SP, Brazil \\ ${ }^{2}$ Department Physical Chemistry, University of Seville, 41012 Seville, Spain \\ (Received 23 July 2013; revised manuscript received 23 January 2014; published 20 February 2014)
}

\begin{abstract}
The appearance of unusually large Au-Au bond distances in linear atomic chains (LACs) of Au nanowires is commonly attributed to the presence of atomic impurities. However, the origin of those contaminants is unknown. We present a study based on density functional theory calculations using quasistatic $(T=0)$ and finite-temperature $a b$ initio molecular-dynamics simulations of a possible route for the formation of atomic impurities in $\mathrm{Au}$ nanowires. This process starts with the adsorption of an $\mathrm{O}_{2}$ molecule followed by a $\mathrm{CO}$ molecule on Au LACs, leading to the formation of an intermediate $\mathrm{O}_{2} \mathrm{CO}$ complex. Upon thermal activation at finite temperatures, the complex is able to proceed to oxidation forming a $\mathrm{CO}_{2}$ molecule and leaving an atomic $\mathrm{O}$ impurity in the Au LAC.
\end{abstract}

DOI: 10.1103/PhysRevB.89.085417

PACS number(s): 31.15.A-, 62.23.Hj, 82.30.-b, 87.15.hp

\section{INTRODUCTION}

One of the very exciting achievements of nanoscience, at the turn of the 20th century, was the fabrication of very thin suspended gold nanowires with interesting properties [1]. Experiments in the subsequent years showed the production of thin nanowires (NWs) as thin as a linear atomic chain (LAC) with 4-10 atoms [2-4]. Theory and simulations also produced the evolution of thin NWs showing the formation of LACs [5-8]. These nanostructures present avenues of research with interesting possibilities as nanocircuits, filters, and catalysts.

The observation of large bond distances in LAC NWs, around $3.6 \AA$, intrigued researchers $[1,2,4]$. Several computational studies on Au NWs failed to find such long bond lengths in pure Au NWs [5,6,8]. Experimentalists and theoreticians started to look for foreign agents to explain such large bond lengths, and light atomic impurities were considered to be the reason for the large distances. Electron microscopy, used to image Au NWs, was unable to "see" these small atoms due experimental difficulties of contrast. In the other front, theoreticians were able to ask and answer these questions with the study of the effect of impurities in the LAC of these NWs [9-12]. This line of research has contributed interesting effects, such as the case of oxygen $(\mathrm{O})$ atomic impurities, proposed as clamps that aid the production of longer LACs in Au NWs [13]. Theoretical predictions [13] were experimentally verified $[3,4]$. The reason for the consideration of impurities comes from the fact that even in ultrahigh vacuum used in the experiments, the chamber always contains molecules such as $\mathrm{O}_{2}, \mathrm{CO}, \mathrm{CO}_{2}$, and $\mathrm{H}_{2} \mathrm{O}$, among others [4]. It might be possible that these molecules would contribute somehow to the formation of the single atom impurities. One question remains a mystery: what is the origin of these atomic impurities, and how do they end up in the LAC? The present article gives a possible answer to this experimental question of the formation of single impurities, and for this reason we investigate $\mathrm{Au}$ NWs with LACs of three and four atoms and the routes for the formation of these atomic impurities.

Gold is well known for its nonreactivity, being one of the noblest metals, when in bulk form. As sizes decrease and $\mathrm{Au}$ nanostructures are considered, it can become a good catalyst. Since the work of Haruta [14] that showed CO oxidation catalyzed by supported gold nanoclusters, intense research considered Au nanoclusters as important catalysts [15]. According to Huchings and Haruta [16], the importance of gold as a good catalyst is due to the discovery showing that supported $\mathrm{Au}$ nanoparticles are very active for lowtemperature $\mathrm{CO}$ oxidation [14]. In the search for hydrogen oxidation, they found that composite oxides of gold with some transition metals of group VIII ( $\mathrm{Fe} \mathrm{Co}$, and $\mathrm{Ni}$ ) were much more active for $\mathrm{CO}$ oxidation than for $\mathrm{H}$ oxidation. Later it was found that these gold composites were gold nanoparticles on oxide supports such as $\mathrm{Fe}_{2} \mathrm{O}_{3}, \mathrm{Co}_{3} \mathrm{O}_{4}$, or $\mathrm{NiO}$. Further studies concluded that the nanoparticle size is crucial to the catalytic activity [17]. Another influence on the oxidation is the type of support of the gold nanoclusters, e.g., $\mathrm{TiO}_{2}, \mathrm{Co}_{3} \mathrm{O}_{4}$, and $\mathrm{Fe}_{2} \mathrm{O}_{3}$ [18]. Also, the preparation conditions are very important because they can affect the final shape of the nanoclusters [19]. Theoretical attempts to understand the $\mathrm{CO}$ oxidation mechanisms were undertaken [20-24]. These works tried to find the best scenarios for the reactions to take place. More recently, new and interesting results were published in the case of adsorption of $\mathrm{O}_{2}$ in Au clusters supported in $\mathrm{MgO}$ film. This work showed the formation of one-dimensional gold oxide at the perimeter of the Au nanocluster, with new $\mathrm{O}$ states favoring $\mathrm{CO}$ oxidation [25]. Thus, nanoclusters supported on surfaces have long been targeted from experimental and computational techniques since they expose metal atoms in a wide range of geometrical configurations and low coordination numbers. At times the catalytic role played in different processes has been elucidated, but there are still open questions in this area of research.

Small molecules that exist in the atmosphere, such as $\mathrm{H}_{2}$, $\mathrm{O}_{2}, \mathrm{CO}, \mathrm{CO}_{2}$, and $\mathrm{H}_{2} \mathrm{O}$, do not react with $\mathrm{Au}$. Au nanostructures, on the other hand, exhibit reactivity, and Au nanoclusters have been extensively studied in the role of catalysts. Many experiments on Au NWs, such as high-resolution transmission electron microscopy (HRTEM) experiments, work in ultrahigh vacuum, but even in these clean environments, the chamber always contains some of these small molecules [4]. This fact, along with the reactivity of $\mathrm{Au}$ nanostructures other than nanowires, was the reason for the study of the effect of $\mathrm{O}_{2}$ and $\mathrm{CO}$ molecules in the gas phase approaching the Au LAC. The present systems, namely Au NWs, offer opportunities 


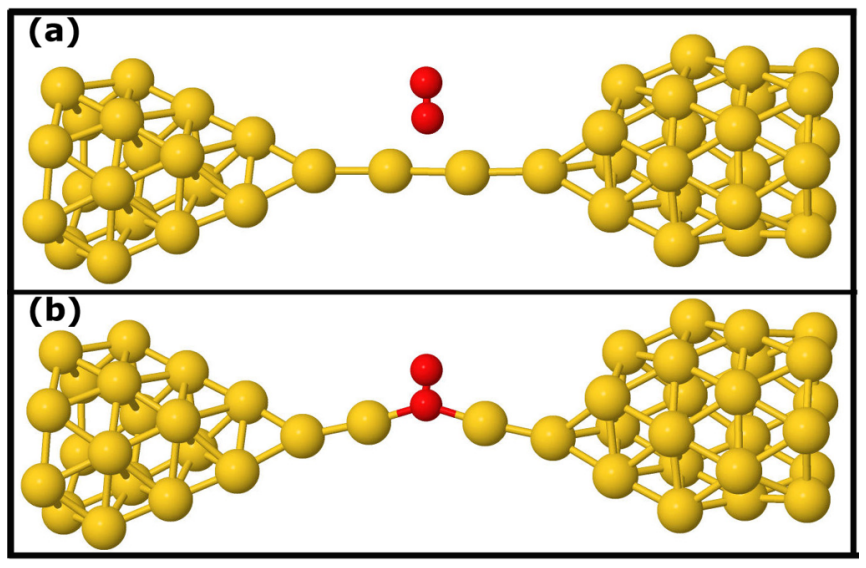

FIG. 1. (Color online) Formation of the Au- $\mathrm{O}_{2}$ complex. (a) $\mathrm{Au}$ pure nanowire with a four-atom linear chain and an $\mathrm{O}_{2}$ molecule in the gas phase nearby. (b) The final stable nanowire with the $\mathrm{Au}-\mathrm{O}_{2}-\mathrm{Au}$ complex.

in this regard, since the reactions studied occur at the linear atomic chain of the NW (see Fig. 1), where LAC atoms are very low coordinated (two Au neighbors) and it is possible to control their bond lengths in experiments such as mechanically controllable breaking junctions (MCBJ) [26]. This control is in fact an important factor for the $\mathrm{O}_{2}$ reaction that in the present case can be driven by mechanical or thermal effects, as will be explained later. Although this work is not aimed at developing a new catalytic process, it presents a pathway involving the $\mathrm{CO}$ oxidation promoted by Au LACs, causing the insertion of single impurities in these LACs and therefore explaining the origin of the unusual large distances.

This paper presents a possible route to the formation of a single atomic impurity in Au NWs. To address this problem, NWs with LACs of four atoms are used. The reason is that they correspond to the initial situation encountered in the experiments [4], where it appears that pure Au NWs with chains of around four atoms may adsorb impurities such as $\mathrm{O}$, which was shown to help the formation of longer LACs [13].

Section II discusses the methodology employed to perform the present study. Then, Sec. III uses the methodology to discuss a chemical route that leads to the formation of an atomic impurity in the Au LAC. This is the case for the formation of an $\mathrm{O}$ impurity, which is of particular interest since $\mathrm{O}$ was previously singled out as an agent that helps the formation of longer Au LACs $[3,4,13]$. This article details this process and lifts the mystery of the origin of the $\mathrm{O}$ atomic impurity in Au-LAC NWs, providing an oxidation route facilitated by the catalytic contribution of the Au-LAC. Finally, Sec. IV presents the conclusions of this work, along with some possible applications of this process.

\section{METHODS}

Our results are based on DFT calculations [27,28]. All calculations used the generalized gradient approximation (GGA) functional under the Perdew-Burke-Ernzerhof (PBE) implementation [29] and norm-conserving pseudopotentials [30]. The pseudopotentials included the $2 s$ and $2 p$ orbitals for $\mathrm{O}$ and $\mathrm{C}$ and $5 d$ and $6 s$ for $\mathrm{Au}[31,32]$, using the SIESTA code.

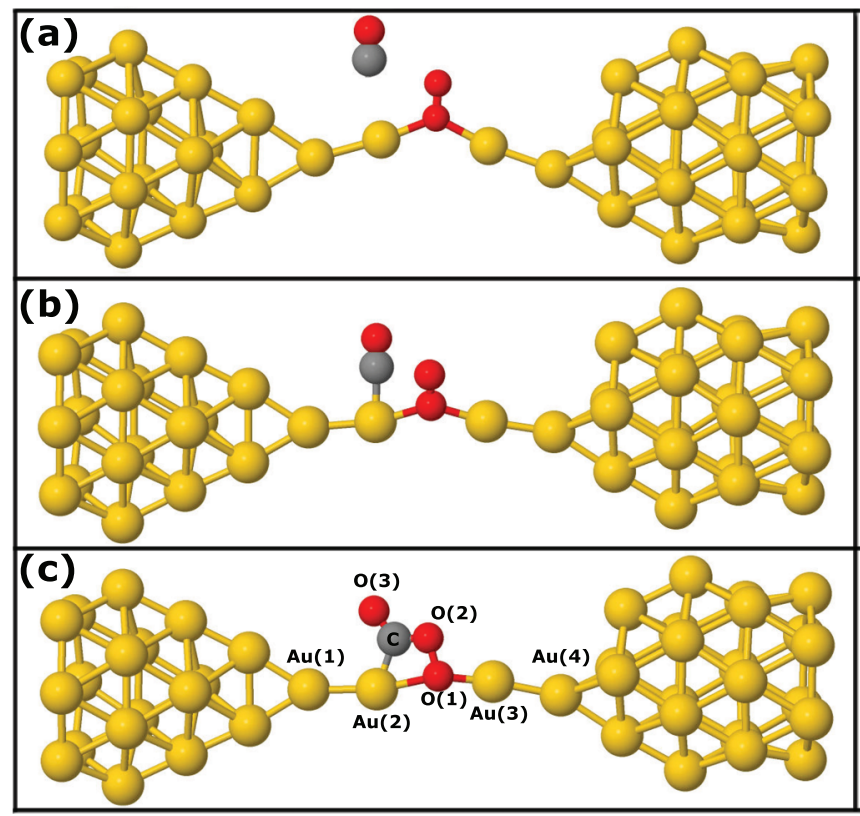

FIG. 2. (Color online) Formation of the Au- $\mathrm{O}_{2} \mathrm{CO}$ complex. (a) The $\mathrm{O}_{2}$ molecule with one $\mathrm{O}$ atom attached to two Au LAC atoms and also a $\mathrm{CO}$ molecule nearby. (b) The $\mathrm{CO}$ molecule adsorbing to one $\mathrm{Au}$ atom $[\mathrm{Au}(2)]$ that has one $\mathrm{Au}-\mathrm{O}$ bond. (c) The final stable $\mathrm{Au}-\mathrm{O}_{2} \mathrm{CO}$ complex.

The calculations used numerical orbitals as basis sets [33]; all atoms were described by a split valence double-zeta basis with polarization function (DZP). A supercell was defined with periodic boundary conditions to define a chain geometry, including a vacuum space of $30 \times 30 \AA$ in the perpendicular directions to the chain to avoid interaction between the NW and its images. The Brillouin zone sampling used the Monkhorst and Pack $k$-points (eight points along the NW direction) [34]. The $T=0$ electronic structure calculations were performed using the conjugate gradient $(\mathrm{CG})$ method, in which all force components were smaller than $0.007 \mathrm{eV} / \AA$.

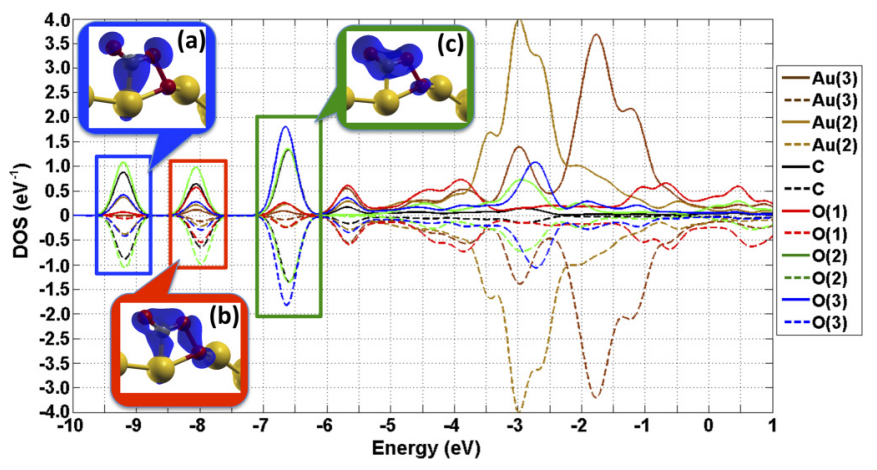

FIG. 3. (Color online) Density of states (DOS) of the $\mathrm{O}_{2} \mathrm{CO}$ complex. Insets are representative total electron density for the $\mathrm{O}_{2} \mathrm{CO}$ complex at specific DOS intervals corresponding to (a) $\sigma$ donation from the $\mathrm{C}$ to the metal, (b) $\pi$ backdonation from $\mathrm{Au} d$ orbitals to the empty $\mathrm{C} p$ orbitals, and (c) $\sigma$ bonds between $\mathrm{C}$ and $\mathrm{O}$ atoms. Isosurfaces are of 0.01 electrons/(Bohr) ${ }^{3}$. Continuum lines denote spin $\alpha$ and dashed lines denote spin $\beta$. 


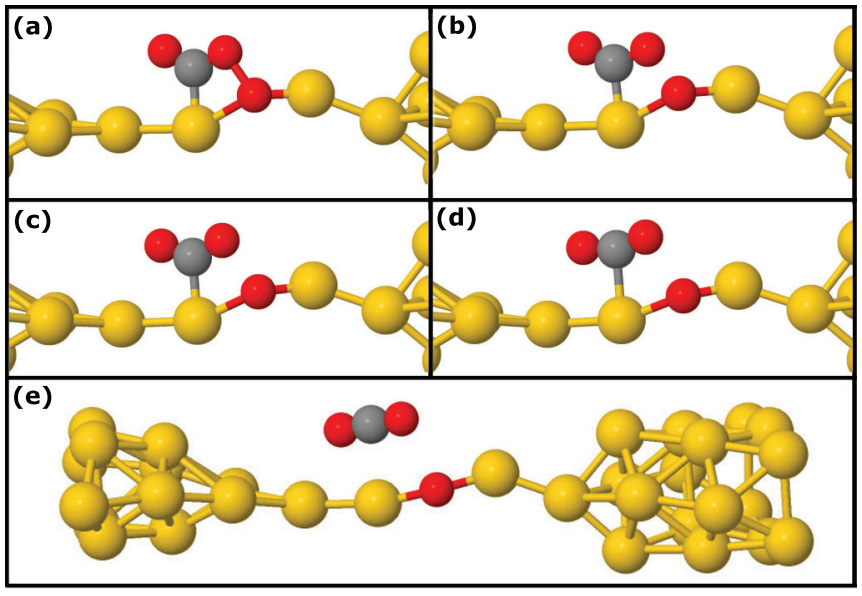

FIG. 4. (Color online) Formation of a $\mathrm{CO}_{2}$ molecule that goes into the gas phase, leaving an atomic $\mathrm{O}$ in the Au NW. (a) The stable complex at an intermediate stage. (b) Breaking of the $\mathrm{O}_{2}$ bond, the starting of the formation of the $\mathrm{CO}_{2}$ molecule. (c) The Au-C distance increases and the $\mathrm{CO}_{2}$ opens its angle. (d) Breaking of the Au-C bond with the formation of a gas phase $\mathrm{CO}_{2}$. (e) $\mathrm{Au} \mathrm{NW}$ with $\mathrm{O}$ atomic impurity and $\mathrm{CO}_{2}$ in gas phase.

The confining energy shift was $0.07 \mathrm{eV}$. The cutoff for the grid integration was 300 Ry for the CG calculations and 250 Ry for the molecular-dynamics simulations. Ab initio molecular dynamics (AIMD) as implemented in SIESTA was used with a procedure similar to previous works [35,36]. The AIMD simulations were performed in the microcanonical ensemble with rescaling of temperature for a target temperature of $500 \mathrm{~K}$ [36]. The equations of motion were integrated by the velocity Verlet algorithm with a time step of 1 fs. The system was thermalized at $300 \mathrm{MD}$ steps (not shown in the figures). The $\mathrm{Au}$ NWs with LACs of four atoms had 44 atoms for the CG calculations. The AIMD calculations of the evolution of the $\mathrm{Au}-\mathrm{O}_{2} \mathrm{CO}$ complex were done using a system containing $32 \mathrm{Au}$ atoms. The two most external Au rings were removed to allow less time-demanding simulations as previously reported [36]. Tests showed that the behaviors of both systems are very similar. These structures came from previous works $[5,6,13]$. All snapshots presented in Figs. 1, 2, and 4 were produced using the open-source code JMOL [37].

\section{RESULTS}

The interaction of $\mathrm{O}_{2}$ molecules with Au structures, namely $\mathrm{Au}$ nanoclusters, free or supported on surfaces, has been studied extensively but is not fully understood yet. In this work, we aim to gain insight into a different system. We focus on the $\mathrm{O}_{2}$ interaction with thin $\mathrm{Au}$ NWs as a precursor for the formation of atomic $\mathrm{O}$ impurities that have been reported previously and theoretically studied. To find these sites, $\mathrm{O}_{2}$ molecules were left at the vicinity of the Au NW. To achieve this goal, we considered Au NWs with linear atomic chains (LACs) of three and four atoms, and $\mathrm{O}_{2}$ molecules at different positions close to the NW, both the tips and the atomic chain. In this work, only NWs with four atoms in the LAC are discussed. Figure 1(a) shows one of these NWs in which the bond length of the Au LAC atoms is on average $2.9 \AA$. Many studies of the approach of an $\mathrm{O}_{2}$ molecule were attempted, using both $\mathrm{CG}$ and AIMD. While $\mathrm{O}_{2}$ attached to Au atoms at the tips, in the chain the stable position was between two Au atoms, bridging the bond and forming $\mathrm{Au}-\mathrm{O}-\mathrm{Au}$ (with the second $\mathrm{O}$ sticking out of the structure) or Au-O-O-Au structures. To find these sites, $\mathrm{O}_{2}$ molecules were left at the vicinity of the $\mathrm{Au} \mathrm{NW}$. In the CG process, initially, $\mathrm{O}_{2}$ molecules were placed $2.0 \AA$ above the middle point of an $\mathrm{Au}-\mathrm{Au}$ bond or above one $\mathrm{Au}$ atom and then the structure was allowed to relax. The most interesting cases were the $\mathrm{O}_{2}$ adsorptions at the central point of the four atom LAC. Figure 1 shows the $\mathrm{NW}$ and the $\mathrm{O}_{2}$ in the gas phase (frame 1) and the $\mathrm{O}_{2}$ inserted in the central Au-Au LAC bond (frame 2). The NW was considered with different degrees of stress, which resulted in different $\mathrm{Au}-\mathrm{Au}$ bond distances for the LAC atoms. As a result, many outcomes occurred. We found that NWs with little tension ( $\mathrm{Au}-\mathrm{Au}$ average distances of around $2.6 \AA$ ) do not adsorb $\mathrm{O}_{2}$ molecules, presumably due to a lack of space in the Au-Au bonds. If the LAC is too stressed ( $\mathrm{Au}$-Au average distances longer than $2.9 \AA$ ), the $\mathrm{O}_{2}$ molecule is inserted with both atoms, forming a peroxy structure Au-O-O-Au. The most interesting case is when the stress is moderate and $\mathrm{Au}-\mathrm{Au}$ average distances are in the range of 2.7-2.9 $\AA$. In those cases, the $\mathrm{O}_{2}$ molecule is adsorbed into the LAC but only one $\mathrm{O}$ atom bonds to the $\mathrm{Au}-\mathrm{Au}$ chain forming Au-O-Au bonds [Fig. 1(b) and also Fig. 2(a)]. This result was found using both CG calculations and AIMD simulations at $T=300 \mathrm{~K}$. Figure 2(a) shows the final stage of the AIMD evolution of the $\mathrm{O}_{2}$ molecule. A film (VIDEO-1 provided in the supplemental material [38]) illustrates the $\mathrm{O}_{2}$ molecule approaching from the gas phase and the adsorption process to the Au LAC leading to the formation of a stable structure, which is the precursor of the next stage.

The initial process considered was the adsorption of an $\mathrm{O}_{2}$ molecule on the LAC (Fig. 1), which turned out to be favorable $(-0.89 \mathrm{eV})$. Further adsorption of a CO molecule is also favored $(-1.92 \mathrm{eV})$, and it led to the formation of an $\mathrm{O}_{2} \mathrm{CO}$ complex that upon activation proceeds to the oxidation and release of a $\mathrm{CO}_{2}$ molecule.

The $\mathrm{O}_{2}$ adsorption energy varies quite significantly with stress, which changes the average $\mathrm{Au}-\mathrm{Au}$ LAC bond length. For average $\mathrm{Au}-\mathrm{Au}$ distances of $2.7 \AA$, it is $-0.41 \mathrm{eV}$ (which is comparable with values reported by Yoon et al. [39]), while for $\mathrm{Au}-\mathrm{Au}$ distances of $2.9 \AA$, it is $-0.89 \mathrm{eV}$. This fact illustrates significant differences from nanowires with respect to surfaces and clusters in which the atom-atom distances are more restricted because of the three-dimensional arrangement. Thus, in nanowires those distances can be controlled by stress, opening new adsorption scenarios. In fact, CMBJ experiments can control the stress on the NW. Our simulations mimic this feature available in thin metal NWs.

A CO molecule can also be adsorbed on the LAC next to the $\mathrm{O}_{2}$-LAC forming a stable $\mathrm{O}_{2} \mathrm{CO}$ complex. Upon activation mediated by mechanical deformations or thermal fluctuations, this complex follows an oxidation pathway forming a $\mathrm{CO}_{2}$ molecule and leaving a single $\mathrm{O}$ atom inserted in the LAC. It is well known that the oxidation of $\mathrm{CO}$ is a process hindered by a high-energy barrier, and only catalytic activity allows that process to take place [40]. Most of the pathways proposed involve the presence of $\mathrm{O}$ adatoms which interact with the adsorbed $\mathrm{CO}$ forming the $\mathrm{CO}_{2}$ [21]. It has also been 
suggested that this oxidation reaction occurs in Au steps and $\mathrm{Au}$ clusters supported on $\mathrm{MgO}$, through the formation of a metastable intermediate $\mathrm{O}_{2} \mathrm{CO}$ complex [21,23] similar to the ones observed in our studies.

The stable structure depicted in Fig. 2(c) has some interesting features worth mentioning. It forms an $\alpha$ angle of $106^{\circ}$ [see also the inset in Fig. 5(a)]. CG calculations with the complex rotated to an angle of $100.8^{\circ}$, which increased the $\mathrm{O}(1)-\mathrm{O}(2)$ bond, made the complex unstable and led to the formation of a $\mathrm{CO}_{2}$ molecule, leaving an $\mathrm{O}$ atomic impurity adsorbed in the LAC. If the energy barrier for this process is modest, it could occur due to thermal fluctuations. To verify this possibility, we performed AIMD simulations at $500 \mathrm{~K}$. Therefore, the stable $\mathrm{O}_{2} \mathrm{CO}$ complex obtained from CG calculations [Fig. 2(c)] was taken as input to AIMD simulations. After thermalization, the evolution of the structure as observed was indeed a concerted process that occurred in many different simulations. We shall describe in detail one of these processes at temperature $500 \mathrm{~K}$.

The formation of the complex assisted by the NW occurs with no energy barrier when a $\mathrm{CO}$ molecule approaches the $\mathrm{O}_{2}$-LAC [Figs. 1(b) and 1(c)]. The adsorption energy for this process is $-1.92 \mathrm{eV}$. A typical density of states (DOS) of the complex showing the contribution of the most important atoms in the complex formation is depicted in Fig. 3. Details of bond formation are given in the insets (a), (b), and (c), where isosurfaces of the most important contribution to the bonding are presented.

The CO fragment in the $\mathrm{O}_{2} \mathrm{CO}$ complex [see Fig. 2(c)] interacts with $\mathrm{Au}(2)$ involving $\sigma$ donation from the $\mathrm{C}$ atom to the metal [Fig. 3, inset (a)] and simultaneously a $\pi$ backdonation from $\mathrm{Au} d$ orbitals to the empty $\mathrm{C} p$ orbitals [inset (b) in Fig. 3]. In the complex, this $\mathrm{Au}(2)-\mathrm{C}$ bond is weakened due to the formation of a new bond between the $\mathrm{C}$ atom and the $\mathrm{O}(2)$ in a characteristic $s p^{2}$ hybridization scheme. This weakens the $\mathrm{Au}(2)-\mathrm{O}(1)$ bond and enlarges the O-O distance by around $0.2 \AA$ of the peroxo bond. Inset (c) in Fig. 3 shows $\sigma \mathrm{C}-\mathrm{O}$ bonds that will evolve to form the $\mathrm{CO}_{2}$ molecule. The electron density contributions to these bonds in the $\mathrm{O}_{2} \mathrm{CO}$ complex can be visualized from representative regions (depicted in colored boxes associated with the insets) in the DOS plot of Fig. 3. The DOS corresponds to the complex structure depicted in Figs. 2(c) and 4(a).

In the AIMD simulation, the $\mathrm{O}_{2} \mathrm{CO}$ complex depicted in Fig. 4(a) stays stable for a period of time. When the $\mathrm{O}(1)-\mathrm{O}(2)$ bond increases, a concerted process starts and the formation of $\mathrm{CO}_{2}$ occurs rapidly. To verify this result, we also performed the AIMD simulation using a Nose-Hoover thermostat (as implemented in the SIESTA code) at the same temperature, and the reaction occurred similarly. In the supplemental material, videos (VIDEO-2, VIDEO-3, VIDEO-4) illustrate the reaction using both AIMD procedures.

Figure 4(b) shows a moment just after the breaking of the $\mathrm{O}(1)-\mathrm{O}(2)$ bond. At this stage, similar to the effect observed in CG, the $\alpha$-angle reached smaller values. The structure started to become unstable, the $\mathrm{O}(1)-\mathrm{O}(2)$ and the $\mathrm{Au}(2)-\mathrm{C}$ distances increased, and the $\mathrm{Au}(2)-\mathrm{O}(1)$ bond was reestablished [Fig. 4(c)]. Next, the $\mathrm{Au}(2)-\mathrm{C}$ bond increases further [Fig. 4(d)], and then the $s p^{2}$ bonds that hold the two O atoms to the $\mathrm{C}$ atom transform into $s p$ bonds as the $\mathrm{Au}(2)-\mathrm{C}$

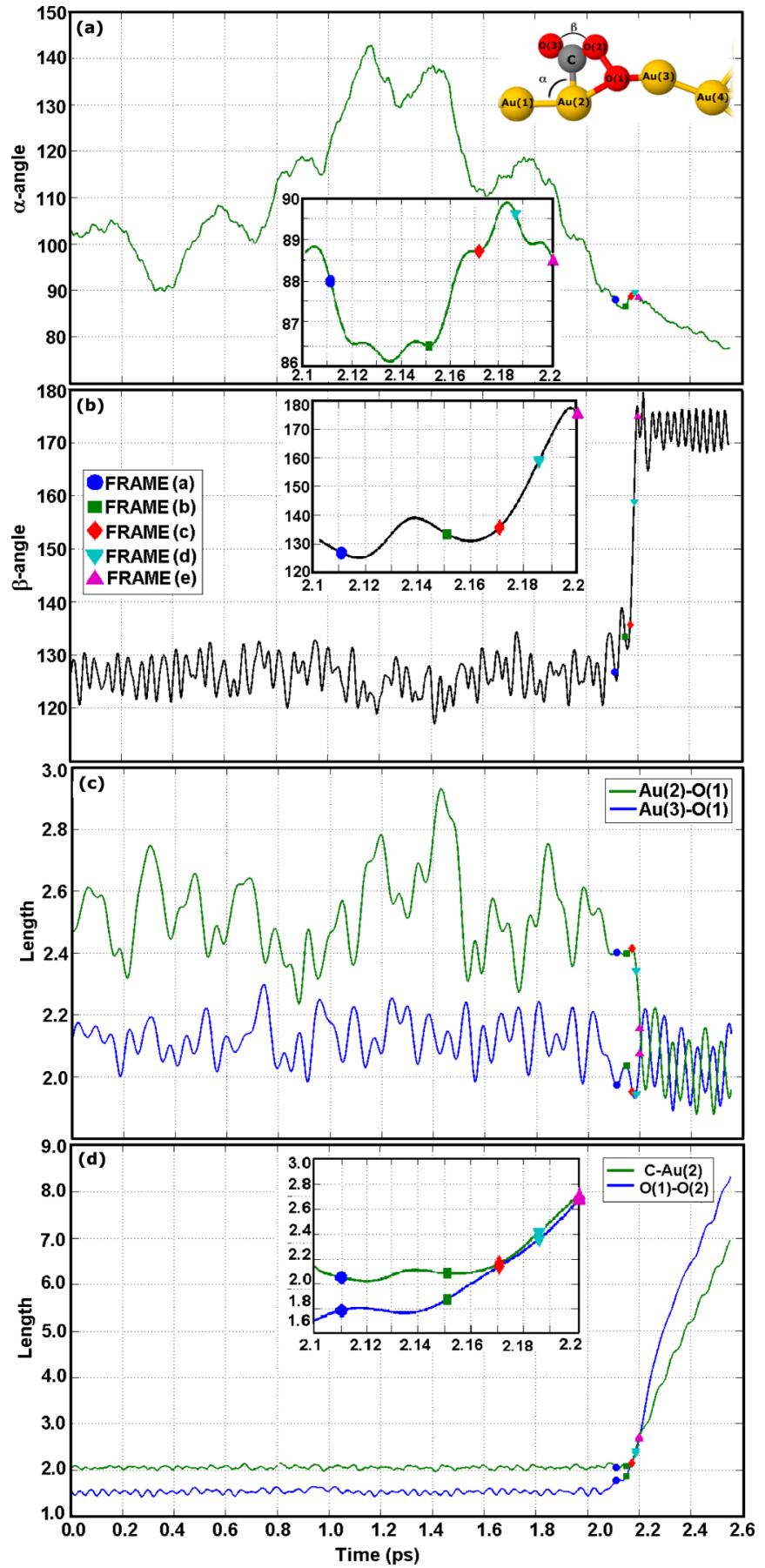

FIG. 5. (Color online) The MD simulation showing the time dependence of some particular bonds and angles. Five special points in colored symbols depict the selected five snapshots of Fig. 2 [named FRAME (a)-(f)]. They are highlighted in the insets. (a) The evolution of the $\alpha$ angle (inset of the NW structure, refers to angles and bonds depicted in the plots). (b) The evolution of the $\beta$ angle. (c) The evolution of the $\mathrm{Au}(2)-\mathrm{O}(1)$ and the $\mathrm{Au}(3)-\mathrm{O}(1)$ bonds. (d) The evolution of the $\mathrm{C}-\mathrm{Au}(2)$ and the $\mathrm{O}(1)-\mathrm{O}(2)$ bonds.

bond breaks and the O-C-O structure transforms into a $\mathrm{CO}_{2}$ molecule, which goes into the gas phase as seen in Fig. 4(e).

To prove the above statements, Fig. 5 shows the evolution of some important distances and angles along the AIMD 
simulation. Graph (a) shows the evolution of the $\alpha$-angle. It can be seen that under the fluctuations, this angle can go to smaller values which are not responsible themselves for the rupture of the bonds. In fact, the smaller angles have to be accompanied by other factors such as the weakening and breaking of the $\mathrm{O}(1)-\mathrm{O}(2)$ bond and the strengthening of the $\mathrm{Au}(2)-\mathrm{O}(1)$ bond [see the inset of graph (a)]. The behavior of the $\beta$-angle is depicted in Fig. 5(b), where one can see the opening of this angle toward the value of $180^{\circ}$, which is the angle of the $\mathrm{CO}_{2}$ molecule in the gas phase when the $\mathrm{O}(1)-\mathrm{O}(2)$ bond breaks around frame $\mathrm{C}$. Figure 5(c) shows the behavior of $\mathrm{O}(1)$, the oxygen attached to the Au atoms. As the $\mathrm{O}(2)$ breaks its bond with $\mathrm{O}(1)$, the $\mathrm{Au}(2)-\mathrm{O}(1)$ bond distance shortens and becomes equal to the $\mathrm{Au}(3)-\mathrm{O}(1)$ bond. The average $\mathrm{Au}(2)-\mathrm{O}(1)$ bond distance depends on the stress of the NW. If this bond is larger due to stress, it hinders the occurrence of the reaction. Figure 5(d) shows the behavior of the $\mathrm{O}(1)-\mathrm{O}(2)$ distance, which starts to increase in frame $\mathrm{B}$, just before the increase of the $\mathrm{Au}(2)-\mathrm{C}$ bond at frame $\mathrm{C}$ resulting in its breaking and the formation of a $\mathrm{CO}_{2}$ molecule. Therefore, the $\mathrm{CO}_{2}$ molecule leaves an atomic $\mathrm{O}$ impurity in the LAC NW.

\section{CONCLUSIONS}

In conclusion, we used state-of-the-art computer simulations to perform DFT calculations ( $\mathrm{CG}$ and finite-temperature AIMD) to study the reactivity of thin Au NWs. We presented a possible route for the formation of such atomic impurities through a catalytic oxidation process involving $\mathrm{O}_{2}$ and $\mathrm{CO}$ molecules resulting in a harmless $\mathrm{CO}_{2}$ molecule and one atomic $\mathrm{O}$ impurity inserted into the LAC NW. We show that $\mathrm{O}_{2}$ can be adsorbed on the LAC in different forms, depending on the degree of tension experienced by the NW. One of these situations (discussed in detail in Sec. III) describes a reaction where an $\mathrm{O}_{2}$ molecule attaches only one of its $\mathrm{O}$ atoms to two $\mathrm{Au} \mathrm{LAC}$ atoms. After this event, a $\mathrm{CO}$ molecule, that is nearby, can also attach to one Au LAC atom forming an $\mathrm{O}_{2} \mathrm{CO}$ complex. Then a concerted evolution motion of this complex produces a $\mathrm{CO}_{2}$ molecule that detaches from the LAC leaving a single atomic impurity in the Au LAC. This process might unveil the mystery of the origin of $\mathrm{O}$ atomic impurities in $\mathrm{Au}$ NWs. We hope that this study will prompt interesting experiments and also the search for other routes of chemical reactions that can be catalyzed by $\mathrm{Au}$ NWs.

\section{ACKNOWLEDGMENTS}

M.A.S. would like to thank CAPES for providing a grant to support a stay at the Institute of Physics "Gleb Wataghin" in UNICAMP. A.P.F.N. would like to thank CAPES for a Ph.D grant. This research had financial support from FAPESP (Project: 2010/16970-0), CNPq (Grant No. 304068/20110), CAPES (REDE NANOBIOTEC-BRASIL/ 04/2008), and FAEPEX-UNICAMP. The calculations were performed at the National Center for High Performance Computing in Sao Paulo (CENAPAD-SP).
[1] H. Ohnishi, Y. Kondo, and K. Takayanagi, Nature (London) 395, 780 (1998).

[2] V. Rodrigues and D. Ugarte, Phys. Rev. B 63, 073405 (2001).

[3] W. H. A. Thijssen, D. Marjenburgh, R. H. Bremmer, and J. M. van Ruitenbeek, Phys. Rev. Lett. 96, 026806 (2006).

[4] T. Kizuka, Phys. Rev. B 77, 155401 (2008).

[5] E. Z. da Silva, A. J. R. da Silva, and A. Fazzio, Phys. Rev. Lett. 87, 256102 (2001).

[6] E. Z. da Silva, F. D. Novaes, A. J. R. da Silva, and A. Fazzio, Phys. Rev. B 69, 115411 (2004).

[7] E. P. M. Amorim and E. Z. da Silva, Phys. Rev. Lett. 101, 125502 (2008).

[8] E. Anglada, J. A. Torres, F. Yndurain, and J. M. Soler, Phys. Rev. Lett. 98, 096102 (2007).

[9] F. D. Novaes, A. J. R. da Silva, E. Z. da Silva, and A. Fazzio, Phys. Rev. Lett. 90, 036101 (2003).

[10] N. V. Skorodumova and S. I. Simak, Phys. Rev. B 67, 121404(R) (2003).

[11] R. N. Barnett, H. Hakkinen, A. G. Scherbakov, and U. Landman, Nanolett. 4, 1845 (2004).

[12] F. D. Novaes, E. Z. da Silva, A. J. R. da Silva, and A. Fazzio, Appl. Phys. A 81, 1551 (2005).

[13] F. D. Novaes, A. J. R. da Silva, E. Z. da Silva, and A. Fazzio, Phys. Rev. Lett. 96, 016104 (2006).

[14] M. Haruta, T. Kobayashi, H. Sano, and N. Yamada, Chem. Lett. 2, 405 (1987).

[15] R. Coquet, K. L. Howard, and D. J. Willock, Chem. Soc. Rev. 37, 2046 (2008).
[16] G. Huchings and M. Haruta, Appl. Catal. A 291, 2 (2005).

[17] M. Valden, X. Lai, and D. W. Goodman, Science 281, 1647 (1998).

[18] M. M. Schubert, S. Hackenberg, A. C. van Veen, M. Muhler, V. Plzak, and R. J. Behm, J. Catal. 197, 113 (2001).

[19] A. Wolf and F. Schuth, Appl. Catal. A 226, 1 (2002).

[20] M. Mavrikakis, P. Stoltze, and J. K. Norskov, Catal. Lett. 64, 101 (2000).

[21] Z.-P. Liu, P. Hu, and A. Alavi, J. Am. Chem. Soc. 124, 14770 (2002).

[22] Z.-P. Liu, X.-Q. Gong, J. Kohanoff, C. Sanchez, and P. Hu, Phys. Rev. Lett. 91, 266102 (2003).

[23] L. M. Molina and B. Hammer, Phys. Rev. Lett. 90, 206102 (2003)

[24] Y. Xu and M. Mavrikakis, J. Phys. Chem. B 107, 9298 (2003).

[25] P. Frondelius, H. Hakkinen, and K. Honkala, Angew. Chem. Int Ed. 49, 7913 (2010).

[26] R. H. M. Smit, Y. Noat, C. Untiedt, N. D. Lang, M. C. van Hemert, and J. M. van Ruitenbeek, Nature (London) 419, 906 (2002).

[27] P. Hohenberg and W. Kohn, Phys Rev. 136, B864 (1964).

[28] W. Kohn and L. J. Sham, Phys. Rev. 140, A1133 (1965).

[29] J. P. Perdew, K. Burke, and M. Ernzerhof, Phys. Rev. Lett. 77, 3865 (1996).

[30] N. Troullier and J. L. Martins, Phys. Rev. B 43, 1993 (1991).

[31] P. Ordejón, E. Artacho, and J. M. Soler, Phys. Rev. B 53, R10441 (1996). 
[32] D. Sánchez-Portal, P. Ordejón, E. Artacho, and J. M. Soler, Int. J. Quantum Chem. 65, 453 (1997).

[33] E. Artacho, D. Sanchez-Portal, P. Ordejon, A. Garcia, and J. M. Soler, Phys. Status Solidi 125, 809 (1999).

[34] H. J. Monkhorst and J. D. Pack, Phys. Rev. B 13, 5188 (1976).

[35] E. Hobi Jr., A. J. R. da Silva, F. D. Novaes, E. Z. da Silva, and A. Fazzio, Phys. Rev. Lett. 95, 169601 (2005).

[36] E. Hobi Jr., A. Fazzio, and A. J. R. da Silva, Phys. Rev. Lett. 100, 056104 (2008).
[37] Jmol: an open-source Java viewer for chemical structures in 3D. http://www.jmol.org/

[38] See Supplemental Material at http://link.aps.org/supplemental/ 10.1103/PhysRevB.89.085417 to view the videos that illustrate the AIMD simulations.

[39] B. Yoon, H. Hakkinan, and U. Landman, J. Phys. Chem. A 107, 4066 (2003).

[40] G. A. Somorjai, Introduction to Surface Chemistry and Catalysis (Wiley, New York, 1994). 